\title{
Experimental and ab initio molecular dynamics study of hydrogen bond systems in selected crystals
}

\author{
L. M. Malec, M. Z. Brela, K. M. Stadnicka \\ Faculty of Chemistry, the Jagiellonian University in Kraków, Gronostajowa 2, 30-387 Kraków, Poland, \\ malec@chemia.uj.edu.pl
}

Crystal engineering requires precise insight into intermolecular interactions, which results in the crystal symmetry enabling the emergence of desired physical properties [1,2]. Such a process is based on structural and thermodynamic information, and should also consider the possibility of polymorphism or phase transitions of engineered crystals [3,4]. Therefore, the controlled synthesis and development of new multifunctional materials and pharmaceuticals should involve the understanding of the dynamics of their interaction networks. One of the most important and abundant intermolecular interactions in crystalline systems are the hydrogen bonds. Several classifications are available for H-bond description, which are based on geometrical parameters, spectroscopic features and charge density calculations. The analysis of different molecular arrangements formed via H-bonds is crucial to understand the stability of crystal phases and the origins of their physical properties [3-5].

In this investigation, the dynamics of complicated H-bond systems in selected crystals were studied using Born-Oppenheimer molecular dynamics (BOMD) simulations. Ab initio molecular dynamics computations provide on the flight evaluation of atomic force evolution using first-principles DFT calculations at every time step. BOMD simulations enable the characterization of solid-state phase dynamics in several statistical ensembles. The insight into crystal entropy and energy is given by the appropriate ensemble averages. The canonical ensemble (NVT) gives the possibility to study the temperature influence on the molecular motion, elastic properties as well as spectroscopic features. In addition, BOMD features allow considering the influence of anharmonicity and quantum effects at the vibrational spectra of examined materials.

In our computations, different cluster sizes were used for investigated H-bonded systems. The system dynamics were studied at different temperatures mainly in the NVT ensemble. Time and space correlations between molecular motions were analysed through the detailed study of interaction network changes along the obtained trajectories. The power spectra were used to investigate the spectroscopic features and the dynamics of considered H-bond systems. Additionally, the structural analysis based on X-ray diffraction experiments was performed, including H-bond propensities and coordination scores. These methods were used to assess the likelihood of specific H-bond formation, and the efficiency of entire H-bond systems according to donor and acceptor expected saturation.

[1] Tiekink, E. R. T., Vittal, J., Zaworotko, M., Ed. (2010). Organic Crystal Engineering: Frontiers in Crystal Engineering. Chichester: John Wiley \& Sons, Ltd.

[2] Nangia, A. K. \& Desiraju, G. R., (2019). Angew. Chem. Int. Ed. 58, 4100.

[3] Bernstein, J., Davey, R. J. \& Henck, J.-O., (1999). Angew. Chem. Int. Ed. 38, 3440.

[4] Price, S. L. (2013). Acta Crystallogr. B69, 313.

[5] Aakeröy, C. B., Forbes, S. \& Desper, J., (2014). CrystEngComm. 16, 5870.

Keywords: hydrogen bonds; molecular dynamics; crystal engineering

Presented computations were performed using PL-Grid Infrastructure and resources provided by ACC Cyfronet AGH. The research was supported by the Polish National Science Centre, project PRELUDIUM 15 number 2018/29/N/ST3/00703 "Study of dynamics in the interaction networks of selected co-crystals". 